\title{
Waterhouse-Friderichsen syndrome complicating primary biliary sepsis due to Pasteurella multocida in a patient with cirrhosis
}

\author{
M Ip, J G C Teo, A F B Cheng
}

\begin{abstract}
Pasteurella multocida is an opportunistic pathogen causing bacteraemia in patients with liver dysfunction. A fulminant case of acute cholecystitis and septicaemia caused by $\boldsymbol{P}$ multocida, complicated by Waterhouse-Friderichsen syndrome without skin haemorrhage, is reported in a previously healthy 64 year old Chinese woman. The patient presented with a six hour history of sudden onset epigastric pain, vomiting, chills, and rigors. A presumptive diagnosis of cholangitis with septicaemic shock was made. Disease progression was rapid and the patient died within eight hours of symptom onset. This case is further proof that skin and mucosal haemorrhages are not an essential feature of Waterhouse-Friderichsen syndrome and this condition should be suspected in all patients presenting with sudden illness and fulminant septicaemia.

(f Clin Pathol 1995;48:775-777)
\end{abstract}

Keywords: Waterhouse-Friderichsen syndrome, cirrhosis, Pasteurella multocida.

Pasteurella multocida, a small Gram negative coccobacillus, causes a variety of infections in humans. The organism is distributed worldwide, occurring as a commensal in the nasopharynx and gastrointestinal tract of many domestic and wild animals. The type of infection seen most frequently in humans is cellulitis following an animal bite. ${ }^{1}$ However, infections may also occur without exposure to animals. It has rarely been reported as a cause of intra-abdominal infection and septicaemia in patients with cirrhosis. ${ }^{23} \mathrm{~A}$ case of Waterhouse-Friderichsen syndrome, without skin haemorrhage, complicating acute cholecystitis and bacteraemia caused by Pasteurella multocida in a patient with hepatitis $B$ associated cirrhosis is reported.

\section{Case report}

A previously healthy 64 year old Chinese woman was admitted with a six hour history of sudden onset epigastric pain, vomiting, chills, and rigors. There was no history of recent exposure to animals or invasive medical procedures. On admission, she was jaundiced, febrile $\left(39.5^{\circ} \mathrm{C}\right)$ and hypotensive (systolic pressure $70 \mathrm{mmHg}$ and central venous pressure $5 \mathrm{~cm} \mathrm{H} \mathrm{H}_{2} \mathrm{O}$ ). The patient was tender in the right upper abdominal quadrant. A presumptive clinical diagnosis of acute cholangitis with septicaemic shock was made. Empirical intravenous antibiotic therapy with ampicillin, cefuroxime and metronidazole was initiated. The patient was resuscitated with intravenous fluids and her blood pressure stabilised.

Laboratory investigations revealed the following: haemoglobin, $12.7 \mathrm{~g} / \mathrm{dl}$; white cell count, $9.2 \times 10^{9} / 1$; platelet count, $11 \times 10^{9} / 1$; normal electrolyte and amylase values; bilirubin, $33 \mu \mathrm{mol} / 1$; alanine aminotransferase, $143 \mathrm{U} / 1$; alkaline phosphatase, $92 \mathrm{U} / 1$; albumin, $15 \mathrm{~g} / \mathrm{l}$; total protein $58 \mathrm{~g} / \mathrm{l}$; prothrombin time, 26.4 seconds; and activated partial thromboplastin time, 88.6 seconds. These findings confirmed disseminated intravascular coagulopathy and impaired liver function.

Emergency endoscopic retrograde cholangiography was planned, but abandoned when the patient sustained a cardiac arrest on arrival at the Radiology Department. She was then transferred to the Intensive Care Unit for further management. The patient sustained a second cardiac arrest and died one and a half hours after admission to hospital. Permission to perform a necropsy was granted.

\section{Pathology}

At necropsy, there were no wounds or localised skin infections, and no skin or mucosal haemorrhages.

Internal examination revealed the classic appearances of Waterhouse-Friderichsen syndrome with massive bilateral adrenal haemorrhages. Microscopic examination revealed haemorrhage within the adrenal cortex and medulla, extending into the periadrenal fat. Multiple visceral petechiae were also found.

Other significant findings were mainly within the hepatobiliary system. The gall bladder was oedematous and haemorrhagic, and contained multiple small calcium bilirubinate stones. The rest of the extrahepatic biliary tree was unremarkable. The liver showed micronodular cirrhosis with severe congestion and haem-

Correspondence to: Dr M Ip. 
orrhage. There was also evidence of portal hypertension, with ascites $(350 \mathrm{ml})$ and congestive splenomegaly $(399 \mathrm{~g})$. There were no oesophageal varices. Histologically, the liver showed sinusoidal congestion and extensive haemorrhage into the fibrous septae separating regenerative nodules. Hepatocytes were positive for hepatitis B surface and core antigens on immunohistochemistry.

\section{Microbiology}

$P$ multocida was isolated from an antemorten blood culture (taken on admission), from a postmortem blood culture and, in pure culture, from bile aspirated from the gall bladder at necropsy. The isolates were non-motile, Gram negative coccobacilli yielding mucoid, grey, convex non-haemolytic colonies on horseblood agar at $37^{\circ} \mathrm{C}$ after overnight incubation. The organisms did not grow on MacConkey agar. They produced acid from glucose and sucrose but not from lactose. They were oxidase and catalase positive, and the API20E system (BioMerieux SA, Marcy l'Etoile, France) identified the three isolates as $P$ multocida (reaction code, 0044504). The antibiotic sensitivity patterns of the three isolates were identical on testing with Stoke's agar disc diffusion method using Mastring-S (Mast Diagnostics, Liverpool, UK) and were sensitive to penicillin, ampicillin, cephalosporins, aminoglycosides, and quinolones.

\section{Discussion}

This is the first documented case of Waterhouse-Friderichsen syndrome complicating $P$ multocida septicaemia. The classic features of Waterhouse-Friderichsen syndrome are sudden onset of illness with rapid progression, manifestation of systemic haemorrhagic, especially in the skin, mucous membranes and serosal surfaces, shock and death within 24 hours, and haemorrhage into both adrenals on postmortem examination. In this instance the absence of skin or mucosal haemorrhages may have confounded the diagnosis. This is the third recorded case of WaterhouseFriderichsen syndrome without skin haemorrhage, the two previous cases occurring in young children with Haemophilus influenzae bacteraemia. ${ }^{4}$

Waterhouse-Friderichsen syndrome is rarely seen in adults and is usually related to disseminated intravascular coagulation in those with bacteraemia. Haemorrhagic destruction of the adrenals leads to primary acute adrenocortical insufficiency and circulatory collapse. Common causative organisms include Neisseria meningitis and $H$ influenzae. Isolated cases of Waterhouse-Friderichsen syndrome have also been reported in association with Capnocytophaga species, DF- $2,{ }^{5}$ Escherichia coli ${ }^{6}$ and Acinetobacter calcoaceticus ${ }^{7}$ bacteraemia. Like $P$ multocida, DF-2 is a Gram negative bacillus occurring as an oral commensal of animals and is an opportunistic pathogen in humans. It is also usually transmitted through animal contact or bites, and typically causes meningitis and septicaemia in immunocompromised patients.
In immunocompromised patients $P$ multocida may cause severe localised infections such as meningitis, pneumonia and intra-abdominal infections. ${ }^{3}$ Reported intra-abdominal infections have included primary bacterial peritonitis in patients with cirrhosis, a few of whom underwent endoscopy, ${ }^{89}$ peritonitis due to a ruptured viscus, intra-abdominal abscesses, and postoperative wound infections. ${ }^{8}$ To our knowledge, this is the first documented case of $P$ multocida causing primary biliary sepsis. Our patient had acute cholecystitis and bacteraemia with $P$ multocida isolated from the bile and blood. The causative organisms of these conditions, however, are usually enteric bacilli or streptococci.

Bacteraemia due to $P$ multocida is relatively infrequent but may also occur in immunocompromised patients, particularly in those with liver dysfunction. Localised sites of infection were present in over half of these reported cases. Raffi et $a l^{3}$ stressed the prognostic importance of the underlying disease, cirrhosis and malignancy being associated with a poorer outcome. In their series these authors reported a mortality rate of $31 \%$ in patients with $P$ multocida bacteraemia, with all fatalities occurring in patients with severe decompensated cirrhosis. Our patient had previously undiagnosed, but histologically confirmed, hepatitis B associated cirrhosis with biochemical evidence of impaired liver function.

There was no documented history of recent exposure to animals or invasive medical procedures in the case reported here. Exposure to animals was absent in $21 \%$ of the cases of $P$ multocida bacteraemia reported in the literature. ${ }^{3}$ Another possibility is that previous colonisation of the upper respiratory and gastrointestinal tract may become apparent in immunocompromised patients or following procedures such as endoscopy.

This case illustrates that $P$ multocida can cause severe infection in immunocompromised patients, particularly in those with cirrhosis. Therefore, $P$ multocida should be considered as a possible cause of bacteraemia in such patients, even if a history of exposure to animals is absent. Skin or mucosal haemorrhages are not an essential feature of WaterhouseFriderichsen syndrome. Development of this catastrophic complication should be suspected in all cases presenting with sudden illness and fulminant septicaemia. Confirmation of adrenal pathology by abdominal ultrasonography $^{10}$ may permit early diagnosis and prompt treatment with glucocorticoid replacement therapy. In our patient, however, the rapid progression of her illness (with death occurring within eight hours of onset of symptoms) was such that the outcome probably could not have been altered, even if Waterhouse-Friderichsen syndrome had been diagnosed premortem.

1 Francis D, Holmes M, Brandon H. Pasteurella multocida infections after domestic animal bites and scratches. fAMA 1975;233:42-5.

2 Jacobson J, Miner P, Duffy O. Pasteurella multocida bacteremia associated with peritonitis and cirrhosis. $A m \mathcal{F}$ Gastroenterol 1977;68:486-91.

3 Raffi F, Barrier J, Baron D, Drugeon H, Nicolas F, Courtieu 
A. Pasteurella multocida bacteraemia: Report of 13 cases over twelve years and review of the literature. Scand 7 Infect Dis 1987;19:385-93.

4 Morrison U, Taylor M, Sheahan DG, Keane CT. Waterhouse-Friderichsen syndrome without purpura due to Haemophilus influenzae group B. Postgrad Med 71985 61:67-8.

5 Chaudhuri AK, Hartley RB, Maddocks AC. WaterhouseFriderichsen syndrome caused by a DF-2 bacterium in splenectomised patient. $\mathcal{f}$ Clin Pathol 1981;34:172-3.

6 Ellert-Zygadlowska J, Trocha H, Tolwinska I. ZespoL Waterhousea i Friderichsena $w$ przebiegu posocznicy E. coli. Wiad Lek 1977;30:1277-80.

7 Ferrara SD, Bertoloni G, Terribile V. Sudden death due to
Waterhouse-Friderichsen syndrome and purulent leptomeningitis caused by Acinetobacter calcoaceticus. $Z$ Rechtsmed 1977;80:73-8.

8 Weber D, Wolfson J, Swartz M, Hooper D. Pasteurella multocida infections-report of 34 cases and review of the literature. Medicine 1984;63:133-54.

9 Brivet F, Guibert M, Barthelemy P, Lepicard A, Naveau S, Dormont J. Pasteurella multocida sepsis after haemorrhagic shock in a cirrhotic patient: Possible role of endoscopic procedures and gastrointestinal translocation. Clin Infect Dis 1994;18:842-3.

10 Sarnaik AP, Sanfilippo DJ, Slovis TL. Ultrasound diagnosis of adrenal hemorrhage in meningococcemia. Pediatr Radio $1988 ; 18: 427-8$

\title{
Microbiological investigation of polyarthralgia
}

\author{
J Wynne Jones, J V S Pether, R W P Frost
}

Public Health

Laboratory,

Musgrove Park

Hospital,

Taunton,

Somerset, TA1 5DB

J Wynne Jones

J V S Pether

$R$ W P Frost

Correspondence to:

Dr J Wynne Jones.

Accepted for publication

21 February 1995

\begin{abstract}
Results of serological investigations on patients with joint pain, arthralgia or polyarthritis were analysed and this information was used to develop a diagnostic algorithm to ensure optimal utilisation of laboratory resources. Accordingly, all cases are now examined for parvovirus IgM, mycoplasma IgM and streptococcal antibodies. Further tests are undertaken by following the algorithm after obtaining supplementary information from a questionnaire. This approach is put forward as a preliminary standard which other laboratories may like to evaluate and develop according to local requirements. ( $f$ Clin Pathol 1995;48:777-779)
\end{abstract}

Keywords: Polyarthralgia, algorithm.

Selection of the appropriate serological investigations in patients with arthralgia often poses difficulty. ${ }^{12}$ To ensure the optimal use of resources and time, it is important that the relevant investigations are chosen from the wide range of available tests.
Tests performed on 140 serum samples submitted for investigation of polyarthralgia

\begin{tabular}{llc}
\hline Investigation & $\begin{array}{l}\text { Number of samples } \\
\text { tested }\end{array}$ & $\begin{array}{l}\text { Number of samples } \\
\text { positive (\%) }\end{array}$ \\
\hline Routine virus screen & 117 & 0 \\
ASO & 117 & $12(10 \cdot 3)$ \\
Monolatex (heterophil antibodies) & 108 & $10(9 \cdot 2)$ \\
Rubella IgM (ELISA) & 110 & 1 \\
Mycoplasma IgM (ELISA) & 101 & $2(1 \cdot 9)$ \\
Parvovirus IgM (radioimmunoassay) & 122 & $35(28 \cdot 7)$ \\
Lyme serology (ELISA) & 18 & 1 \\
\hline
\end{tabular}

Routine viral CFTs include the following antigens: influenza A and B; adenovirus; respiratory syncytial virus; $Q$ fever; and Psittacosis. Serology tests for Leptospira $(\mathrm{n}=3)$, Brucella $(\mathrm{n}=2)$, Yersinia $(\mathrm{n}=2)$, Toxoplasma $(\mathrm{n}=3)$, hepatitis $\mathrm{B}(\mathrm{n}=1)$, and Chlamydia $(\mathrm{n}=2)$ were also Yersinia $(\mathrm{n}=2)$, Toxoplasma $(\mathrm{n}$
performed with negative results.

\section{Methods}

To determine the current practice in our laboratory, the results of the serology performed over the last six months on patients with a clinical history of joint pain were analysed. On the basis of these observations, guidelines for the future investigation of patients with polyarthralgia were prepared.

\section{Results}

The serological findings for the 140 patients studied are shown in the table. An outbreak of parvovirus infection coincided with this study and polyarthritis was attributed to this infection in one quarter of all patients investigated.

Four serum samples had antistreptolysin $\mathrm{O}$ (ASO) titres $>800 \mathrm{units} / \mathrm{ml}$ and DNAse antibodies above 300 units $/ \mathrm{ml}$. Six serum samples had ASO titres between 500 and 800 units $/ \mathrm{ml}$, all indicative of recent streptococcal infection. An additional two serum samples had raised anti-DNAse antibodies with a normal ASO which, in the presence of a compatible clinical history, were also considered suggestive of a streptococcal illness. In total, 12 serum samples exhibited evidence of recent streptococcal infection - that is, $8.6 \%$ of all the patients studied.

The heterophil antibodies detected by the positive monolatex tests were not confirmed as recent Epstein-Barr virus (EBV) infectionsthat is, exhibiting a positive immunofluorescence test for EBV capsid antibody in the presence of a negative enzyme linked immunosorbent assay (ELISA) test for EB nuclear antigen antibody. In eight patients the positive monolatex test appeared to be related to the presence of recent infection with parvovirus, a difficulty that has been reported previously. ${ }^{3}$ 